\title{
Formation of ferromagnetic molecular thin films from blends by annealing
}

\author{
Peter Robaschik ${ }^{1}$, Ye Ma ${ }^{1}$, Salahud Din ${ }^{1,2}$ and Sandrine Heutz ${ }^{* 1}$
}

\author{
Full Research Paper \\ Address: \\ ${ }^{1}$ Department of Materials and London Centre for Nanotechnology, \\ Imperial College London, Exhibition Rd, London SW7 2AZ, United \\ Kingdom and ${ }^{2}$ Kurt J. Lesker Company, Sidney Little Rd, St \\ Leonard's on Sea TN38 9PU, United Kingdom \\ Email: \\ Sandrine Heutz* - s.heutz@imperial.ac.uk \\ * Corresponding author \\ Keywords: \\ co-deposition; molecular spintronics; organic thin films; \\ phthalocyanines; tetracyanoquinodimethane (TCNQ)
}

\author{
Beilstein J. Nanotechnol. 2017, 8, 1469-1475. \\ doi:10.3762/bjnano.8.146 \\ Received: 15 March 2017 \\ Accepted: 20 June 2017 \\ Published: 14 July 2017 \\ This article is part of the Thematic Series "Towards molecular \\ spintronics". \\ Guest Editor: G. Salvan \\ (C) 2017 Robaschik et al.; licensee Beilstein-Institut. \\ License and terms: see end of document.
}

\begin{abstract}
We report on a new approach for the fabrication of ferromagnetic molecular thin films. Co-evaporated films of manganese phthalocyanine $(\mathrm{MnPc})$ and tetracyanoquinodimethane (TCNQ) have been produced by organic molecular beam deposition (OMBD) on rigid (glass, silicon) and flexible (Kapton) substrates kept at room temperature. The MnPc:TCNQ films are found to be entirely amorphous due to the size mismatch of the molecules. However, by annealing while covering the samples highly crystalline MnPc films in the $\beta$-polymorph can be obtained at $60{ }^{\circ} \mathrm{C}$ lower than when starting with pure MnPc films. The resulting films exhibit substantial coercivity $(13 \mathrm{mT})$ at $2 \mathrm{~K}$ and a Curie temperature of $11.5 \mathrm{~K}$.
\end{abstract}

\section{Introduction}

Controlling the structure of molecular thin films is of great interest for their application in optoelectronic and spintronic devices [1,2]. One of the key physical properties determining the performance is crystallinity, which significantly influences electron/hole mobility and magnetic coupling $[3,4]$. We recently reported on an approach to achieve crystalline porphyrazine films, which are amorphous on non-interacting substrates, by depositing the molecules on a 3,4,9,10-perylenetetracarboxylic dianhydride (PTCDA) surface, leading to a transformation from paramagnetic to antiferromagnetic behaviour [5]. Another promising class of molecules are phthalocyanines (Pc) that can be easily incorporated in thin films that exhibit outstanding semiconducting and magnetic properties [6].

Phthalocyanines feature different polymorphs, often defined by the angle formed by the molecule and the stacking axis, $\varphi$, which can be controlled via different temperature treatments. For instance, at room temperature, films commonly adopt the $\alpha$-phase $\left(\varphi=65^{\circ}\right)$ and can be transformed to the thermodynamically stable $\beta$-phase $\left(\varphi=45^{\circ}\right)$ by annealing in vacuum at $330^{\circ} \mathrm{C}$ 
[7-9]. However, such temperatures are too high for most flexible substrates, and therefore limit one of the main fabrication advantages of molecular materials.

The $\beta$-phase is particularly attractive for MnPc, where it leads to ferromagnetism in polycrystalline powders and single crystals $[10,11]$. Due to the arrangement in molecular columns with a stacking angle of $45^{\circ}$ the $\mathrm{Mn}$ ion lies directly underneath a nitrogen atom of the nearest-neighbouring $\mathrm{MnPc}$ molecule. The magnetic interaction has been attributed to superexchange [1113], although more recent results highlight that indirect exchange also contributes to the mechanism [14].

Here we develop a strategy to lower the phase-transition temperature of $\mathrm{MnPc}$ by $60^{\circ} \mathrm{C}$ by blending the MnPc film with TCNQ in the starting films deposited at room temperature. Optical microscopy and X-ray diffraction (XRD) are used to identify the phase transition by investigation of the surface morphology and the structure of the films, while FTIR spectroscopy provides information about the chemical composition and structure of the films. Furthermore superconducting quantum interference device (SQUID) magnetometry measurements reveal the ferromagnetic behaviour of the $\beta-\mathrm{MnPc}$ films, which exhibit remarkable coercivity. The opening of the hysteresis loop is preserved at temperatures up to $10 \mathrm{~K}$, and the Curie temperature as determined by susceptibility measurements is $11.5 \mathrm{~K}$. The combination of new processing methodologies with attractive magnetic properties will have important implications for spintronics.

\section{Results and Discussion Sample preparation}

Blended films comprising MnPc and TCNQ (Figure 1a) have been prepared by organic molecular beam deposition (OMBD). A one-to-one ratio of the desired molecules in the films was obtained with a deposition rate of $0.5 \AA / \mathrm{s}$ and a thickness of

a

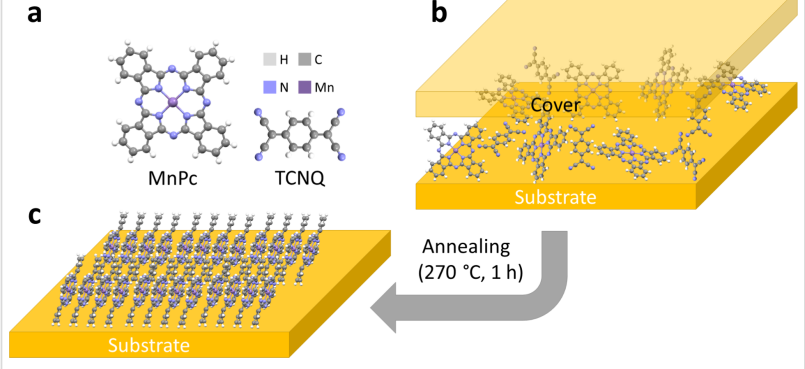

Figure 1: (a) Manganese phthalocyanine $(\mathrm{MnPc})$ and tetracyanoquinodimethane (TCNQ) molecules. (b) Annealing procedure applied to the blended thin films prepared by OMBD. (c) Well-ordered $\beta-M n P c$ film after annealing.
$100 \mathrm{~nm}$ for MnPc. Due to its higher molecular density TCNQ is deposited at a lower rate of $0.22 \AA /$ s leading to a thickness of $44 \mathrm{~nm}$. Hence the total thickness of the blended film amounts to $144 \mathrm{~nm}$.

Figure $1 \mathrm{~b}$ shows the annealing procedure in which the blended film is covered (see Experimental section) without applying any additional force and put inside a tube furnace. Once the tube is pumped down and flushed with nitrogen at a flow rate of $150 \mathrm{sccm}$ for at least one hour the furnace is set to $270{ }^{\circ} \mathrm{C}$ and left dwelling for one hour after the final temperature is reached. During this process the TCNQ molecules can slowly escape the film due to their low sublimation temperature of approximately $100{ }^{\circ} \mathrm{C}[15]$.

We compare these films with neat MnPc films, prepared using sublimation of $\mathrm{MnPc}$ on substrates at room temperature at a rate of $0.5 \AA / \mathrm{s}$ up to a thickness of $100 \mathrm{~nm}$, and subjected to subsequent annealing procedures as for the blends. As will be discussed later we believe that the emerging vacancies in the mixed films generate sufficient free volume around the MnPc molecules for a rearrangement to the thermodynamically stable $\beta$-phase (Figure 1c), which normally forms above $300{ }^{\circ} \mathrm{C}[7,8]$.

\section{Film morphology, structure and composition}

Optical micrographs in Figure 2 reveal the surface morphology of the organic films following different thermal treatments. For comparison the neat $\mathrm{MnPc}$ film deposited on glass kept at room temperature (Figure 2a) is shown with a very smooth surface and a grain size that is below the detection limit of the optical microscope. Annealing at $270{ }^{\circ} \mathrm{C}$ without a cover for one hour (Figure $2 b$ ) does not affect the surface morphology. However, increasing the temperature to $330{ }^{\circ} \mathrm{C}$ and covering the films (Figure 2c) leads to the formation of larger crystallites.

Similar results are obtained for the mixed films (Figure $2 \mathrm{~d}-\mathrm{f}$ ), although annealing at a temperature of $270{ }^{\circ} \mathrm{C}$ already allows the molecules to rearrange into large elongated crystallites. This was observed previously for iron phthalocyanine $(\mathrm{FePc})$ thin films deposited at different substrate temperatures [16]. However, in that case the length of the major axis was found to be $200 \mathrm{~nm}$ at a temperature of $260{ }^{\circ} \mathrm{C}$. The crystallites in our MnPc films reach a size of up to $10 \mu \mathrm{m}$ for their longer axis, which is attractive for high coercivity in magnetism and improved charge transport along the crystallites.

Results from optical microscopy can be refined by X-ray diffraction (XRD). Figure 3a shows the XRD patterns of the as-deposited MnPc:TCNQ film on silicon as well as the films that have been annealed with and without cover. The sample without any heat treatment appears entirely amorphous showing 

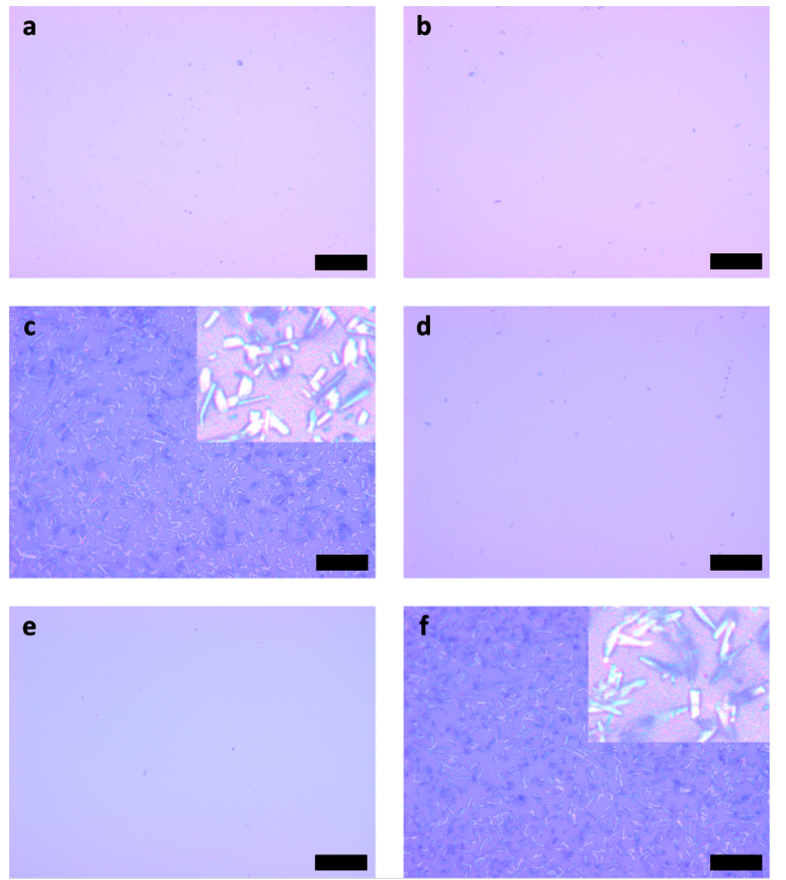

Figure 2: Optical micrographs for molecular thin films grown on glass substrates. (a) $\alpha-M n P c$ film grown at room temperature. (b) MnPc film annealed at $270{ }^{\circ} \mathrm{C}$ without cover. (c) $\beta-\mathrm{MnPc}$ film formed by annealing of the $\alpha-M n P c$ sample at $330^{\circ} \mathrm{C}$ with cover. (d) MnPc:TCNQ film grown at room temperature. (e) MnPc:TCNQ film annealed at $270{ }^{\circ} \mathrm{C}$ without cover. (f) $\beta-\mathrm{MnPc}$ film formed by annealing of a MnPc:TCNQ sample at $270{ }^{\circ} \mathrm{C}$ with cover. The scale bars correspond to a length of $20 \mu \mathrm{m}$ for the images and $5 \mu \mathrm{m}$ for the insets.

no peaks in a range up to $2 \theta=30^{\circ}$, where phthalocyanine fingerprints usually appear [4]. Annealing at a temperature of $270{ }^{\circ} \mathrm{C}$ without a cover also leads to a featureless XRD pattern.
However, as will be shown by FTIR this is due to the absence of any thin film rather than to its amorphous nature.

The annealed MnPc:TCNQ films that were additionally covered, however, show two high intensity peaks at $2 \theta=7.13^{\circ}$ and $9.37^{\circ}$, which correspond to the diffraction from the (001) and $(20-1)$ planes of $\beta$-MnPc $[17,18]$. We note that the $(20-1)$ plane is not usually observed in $\beta-\mathrm{MnPc}$ films obtained by annealing of the $\alpha$-phase and its observation indicates a reduced texture. This is presumably due to the disordered nature of the starting film in this case, in contrast to the case of highly oriented $\alpha$-phase film. The inset of Figure 3 a reveals the sharp nature of both peaks and fits using the Lorentz function result in full width at half maximums (FWHM) of $0.11^{\circ}$ and $0.10^{\circ}$, respectively. From the obtained FWHMs we can estimate the grain dimensions, $\tau$, out of the sample plane by using the Scherrer equation [19]:

$$
\tau=\frac{K \lambda}{\beta \cos (\theta)},
$$

where $K$ is the dimensionless shape factor, $\lambda$ is the wavelength of the X-ray source, $\beta$ is the FWHM and $\theta$ is the Bragg angle. Calculations using $K=1$ give a grain size of $84 \pm 9 \mathrm{~nm}$ for the $\beta-\mathrm{MnPc}$ film, which is in line with the equivalent film thickness of MnPc in the blended film prior to annealing.

By comparison a neat $100 \mathrm{~nm}$ thick MnPc film annealed at $270{ }^{\circ} \mathrm{C}$ for one hour shows only one broad diffraction peak at $2 \theta=7.09^{\circ}$, which most likely combines the contributions of the (100) and (001) planes of the $\alpha$ - and $\beta$-polymorphs, respective-
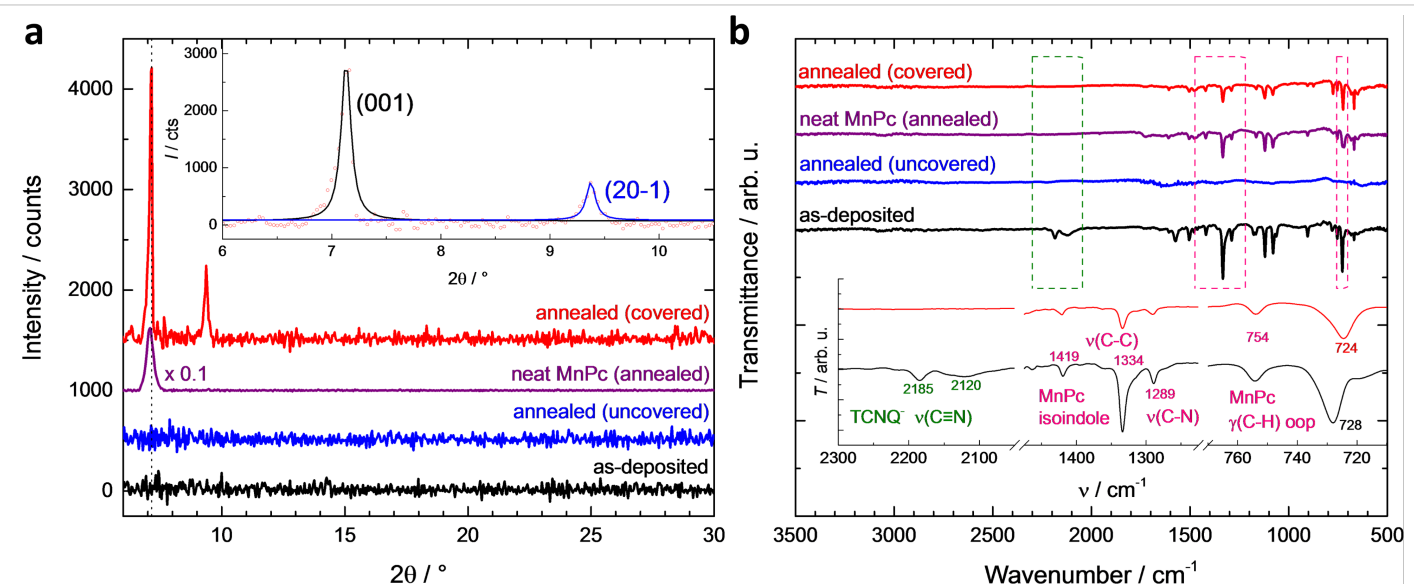

Figure 3: (a) X-ray diffraction patterns. For an as-deposited $144 \mathrm{~nm}$ thick MnPc:TCNQ film on silicon (black) no diffraction peaks are observed. Annealing without cover (blue) leads to similar results, whereas the covered film (red) exhibits two diffraction peaks with high intensity. For comparison the XRD pattern of a neat MnPc film (purple) that was annealed at the same temperature is shown. All films were annealed at a temperature of $270^{\circ} \mathrm{C}$. (b) FTIR spectra for the same films deposited on $\mathrm{KBr}$ substrates. The green frame highlights the range of $2050-2300 \mathrm{~cm}^{-1}$ where the $\mathrm{v}(\mathrm{C} \equiv \mathrm{N})$ stretching peaks for TCNQ appear. The pink frames show the area of the MnPc isoindole vibrations around $1225-1475 \mathrm{~cm}^{-1}$ and the $\mathrm{Y}(\mathrm{C}-\mathrm{H})$ out-ofplane deformation of the MnPc ligand at $710-770 \mathrm{~cm}^{-1}$, respectively. The inset shows specific regions in the mixed films (colour coding as in main graph). 
ly [17,20], assuming that MnPc is isostructural with $\mathrm{CuPc}$. This represents an intermediate case with a partial transformation from $\alpha$ - to $\beta$-phase as shown earlier for metal-free phthalocyanine thin films where Yim et al. proposed a phase-transition mechanism that involves tilting of the $\alpha$-phase molecular columns to form the $\beta$-phase [9]. Therefore, for the neat MnPc film the thermal energy provided is not sufficient to transform the entire film from $\alpha$ - into $\beta$-phase. Due to the combination of both $\alpha$ - and $\beta$-phases, the diffraction peak has been fitted to two Lorentz functions centred at $2 \theta=7.03^{\circ}$ and $7.16^{\circ}$ leading to FWHMs of $0.20^{\circ}$ and $0.21^{\circ}$, respectively. The values correspond to a grain size of $45 \pm 5 \mathrm{~nm}$, which is in agreement with the values of phthalocyanine films previously deposited at room temperature [16,21], correlates with the smooth morphology and is much lower than the value of the $\beta-\mathrm{MnPc}$ films generated from the blended film.

To further investigate the phase transformation we have conducted FTIR spectroscopy measurements (Figure $3 b$ ) on the same films deposited on $\mathrm{KBr}$ substrates. The $v(\mathrm{C} \equiv \mathrm{N})$ stretching peaks of TCNQ for the as-deposited blended film can be found at 2185 and $2120 \mathrm{~cm}^{-1}$, respectively. This finding is in contrast to the peak at $2228 \mathrm{~cm}^{-1}$ for the neutral TCNQ [22] and suggests charge transfer (CT) from the $\mathrm{Mn}$ ion of the phthalocyanines to the TCNQ molecules forming $\mathrm{Mn}^{3+}$ and $\mathrm{TCNQ}^{-}$. Similar CT was previously observed for $\mathrm{MnPc} / \mathrm{F}_{4} \mathrm{TCNQ}$ films by Rückerl and collaborators [23]. Both peaks vanish for every annealed sample proving that all TCNQ molecules sublime during the annealing process. The $v(\mathrm{C}-\mathrm{C})(1429$ and $\left.1334 \mathrm{~cm}^{-1}\right)$ and $v(\mathrm{C}-\mathrm{N})\left(1289 \mathrm{~cm}^{-1}\right)$ vibrations of the $\mathrm{MnPc}$ isoindole [24] are preserved for the annealed neat film and the annealed mixed film with cover. However, for the MnPc:TCNQ blended film that was annealed without cover it seems that both phthalocyanine and TCNQ molecules sublime. Furthermore, for the covered sample, one peak of the $\gamma(\mathrm{C}-\mathrm{H})$ out-of-plane deformation of the MnPc ligand shows a shift from 728 to $724 \mathrm{~cm}^{-1}$ and can be attributed to phase transformation to the $\beta$-phase [25].

We believe that the successful crystallisation of the $\beta$-phase $\mathrm{MnPc}$ at lower temperatures compared to the $\alpha \rightarrow \beta$ phase transition thanks to the blending with sacrificial TCNQ molecules is due to two main factors. Firstly, the presence of TCNQ hinders crystallisation of the MnPc molecules, preventing them from forming strong intermolecular bonds and enabling higher mobility for crystallisation into the thermodynamically stable $\beta$-phase. This is substantiated by the observation in FTIR of the sublimation of MnPc molecules, which are not stabilised by the crystal lattice, in the uncovered blend film at $270{ }^{\circ} \mathrm{C}$ whereas neat $\alpha$-phase films remain on the substrate under the same conditions. Secondly, when the blend film is covered, $\mathrm{MnPc}$ molecules cannot sublime as readily, but TCNQ molecules can diffuse out of the film due to their small size and lower sublimation temperature, creating vacant space which allows the $\mathrm{MnPc}$ molecular columns to arrange into the $\beta$-phase.

So the co-deposition of phthalocyanines with small molecules could allow for the formation of highly oriented molecular films at lower process temperatures. This is crucial for later integration into electronic and spintronic devices, especially on flexible substrates.

\section{Magnetic characterisation}

As expected from optical microscopy and XRD the $\beta$-MnPc film formed by annealing of the MnPc:TCNQ utilising a cover exhibits remarkable magnetic properties. Figure 4 shows magnetic hysteresis loops of a MnPc film on Kapton at different temperatures. A substantial coercivity of $13 \mathrm{mT}$, which coincides with the $15 \mathrm{mT}$ found for $\mathrm{MnPc}$ powder annealed in Ar atmosphere [26], can be detected at $2 \mathrm{~K}$ and an opening of the hysteresis is preserved up to a temperature of $10 \mathrm{~K}$. The magnetisation increases rapidly until a field of $100 \mathrm{mT}$ is reached and is not saturated at the maximum field of $7 \mathrm{~T}$. This is in line with earlier reports on $\beta-\mathrm{MnPc}$ powder and singlecrystal samples $[10,11,27,28]$. However, to our knowledge no reports on the coercivity in $\beta-\mathrm{MnPc}$ thin films from OMBD have been made. The magnetic moment at $7 \mathrm{~T}$ amounts to $2.1 \mu_{\mathrm{B}}$ per MnPc molecules, which is below the expected value of $3 \mu_{\mathrm{B}}$ for a system with $S=3 / 2$. This can be explained by the absence of magnetic saturation at the maximum available field and the reduction of magnetic moment due to crystal field effects [29]. Due to the observation of a reasonable magnetic moment per MnPc we can further confirm that no significant

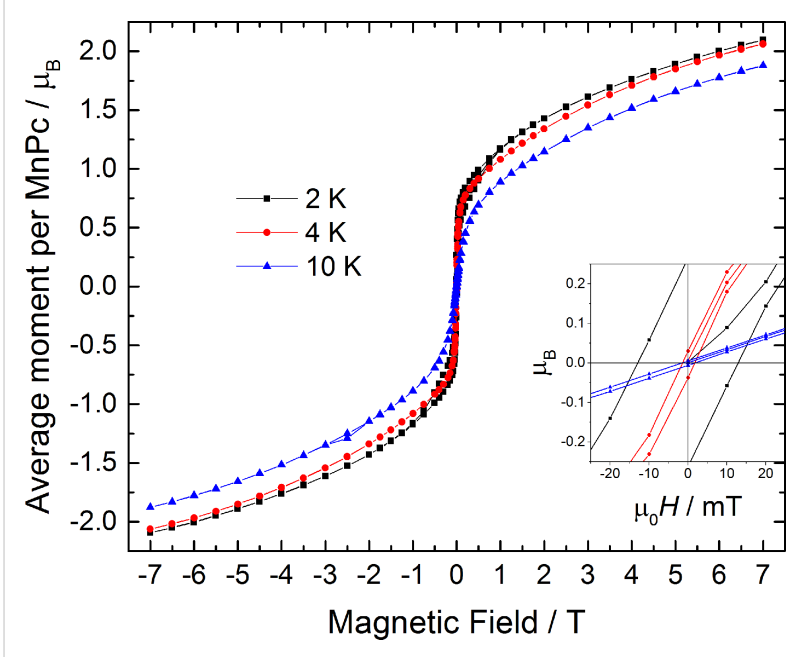

Figure 4: Magnetic hysteresis loops of a MnPc thin film on Kapton obtained from annealing of a MnPc:TCNQ blend. The inset shows that the film exhibits a coercivity of up to $13 \mathrm{mT}$ at $2 \mathrm{~K}$. 
amount of phthalocyanine molecules have been sublimed during the annealing process.

For further analysis the temperature dependence of the magnetisation (Figure 5a) has been measured at fields of 20 and $40 \mathrm{mT}$ for both field cooled (FC) and zero-field cooled (ZFC) protocols. The average magnetic moment per MnPc molecule increases rapidly below $20 \mathrm{~K}$ and shows a bifurcation in both ZFC measurements at $3 \mathrm{~K}$, whereas a steady increase in the FC data can be observed. This finding coincides with reports on DC and $\mathrm{AC}$ susceptibility measurements of pure $\beta-\mathrm{MnPc}$ powder showing a slow relaxation of magnetisation [30,31]. Further studies by Wang and Seehra were able to rule out spin-glass behaviour and found that the zero-field splitting (ZFS) parameter $|D| / k_{\mathrm{B}}=8.3 \mathrm{~K}$ is much larger than the coupling constant $J / k_{\mathrm{B}}=2.6 \mathrm{~K}$ [32]. According to Moriya, in this case $(|D| / J>1)$ long-range ordering is not possible [33], which leads to the assumption that $\beta-\mathrm{MnPc}$ can be treated as a single-chain magnet.

From the temperature-dependent magnetisation values collected at two different fields the inverse differential susceptibility was determined and is shown in Figure 5b. The data follows a linear behaviour at higher temperatures and can be fitted to the Curie-Weiss law in a temperature range from 20 to $50 \mathrm{~K}$. The Curie temperature is found from the intercept with the $y$-axis at $11.5 \mathrm{~K}$ and is slightly higher compared to reported values of 6-10 K for $\beta$-MnPc crystals $[10,11,28,34]$.

\section{Conclusion}

We report on an approach to fabricate ferromagnetic $\beta-\mathrm{MnPc}$ films by annealing of MnPc:TCNQ blends, which reduces the process temperature by $60{ }^{\circ} \mathrm{C}$ compared to the preparation pro- cedure starting from a neat $\mathrm{MnPc}$ film. The resulting films form large elongated crystallites with their long axis measuring up to $10 \mu \mathrm{m}$. X-ray diffraction studies show that the out-of-plane dimensions of the grains $(84 \pm 9 \mathrm{~nm})$ are comparable to the target film thickness of $100 \mathrm{~nm}$, which means that no grain boundaries parallel to the substrate plane are expected. Magnetic measurements reveal remarkable ferromagnetic properties with a substantial coercivity of up to $13 \mathrm{mT}$ at $2 \mathrm{~K}$ and a Curie temperature of $11.5 \mathrm{~K}$. These findings are significant for a future implementation of magnetic molecular thin films in spintronic devices, especially on flexible substrates that are commonly sensitive to high process temperatures. We anticipate that the methodology for reducing phase-transition temperatures through the blending strategy can be generalised to a wide range of systems, provided the sacrificial molecules used in the blend reduce intermolecular interactions and have a sublimation point below that of the molecule of interest.

\section{Experimental}

Organic thin films have been grown using a SPECTROS deposition chamber by Kurt J. Lesker Company with a base pressure of $3 \times 10^{-7}$ mbar. During the co-deposition in total three quartz crystal microbalances (QCM) were utilised to achieve a one-to-one ratio of the molecules. Two QCMs monitored the deposition rate of each organic source and the combined rate was confirmed by a third QCM next to the substrates.

The annealing procedure was carried out in a furnace (Carbolite HZS-12/900E) fitted with a $150 \mathrm{~cm}$ long quartz tube $(3 \mathrm{~cm}$ outer diameter). For the covered samples the films on glass and silicon substrates were sandwiched between microscope slides, whereas the Kapton samples were covered by another Kapton sheet. The tube was pumped down to $1 \times 10^{-1}$ mbar followed
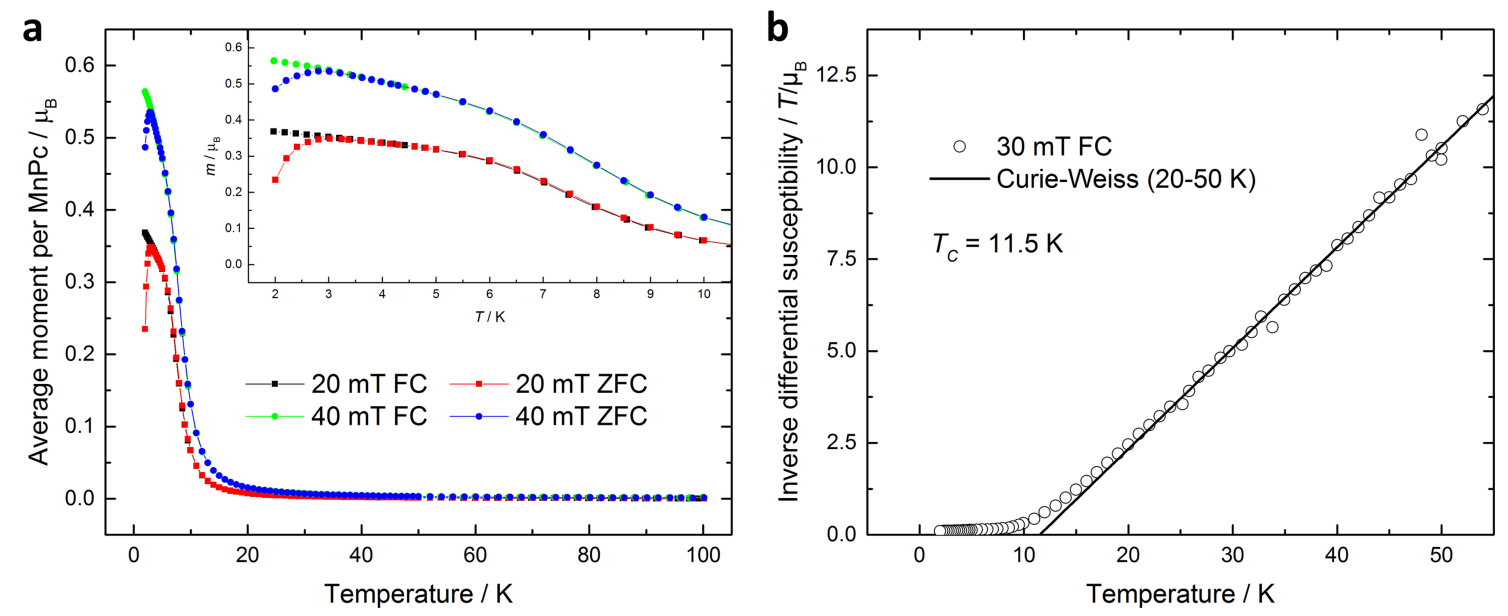

Figure 5: (a) Magnetisation as a function of the temperature at fields of $20 \mathrm{mT}$ and $40 \mathrm{mT}$ using both FC and ZFC protocols. (b) Inverse differential susceptibility calculated from the magnetisation measurements showing a Curie temperature of $11.5 \mathrm{~K}$. 
by nitrogen flushing at a flow rate of $150 \mathrm{sccm}$ for at least one hour. After flushing the nitrogen flow was kept at the same rate leading to a pressure of approximately $25 \mathrm{mbar}$. All three zones of the furnace were set up to $270{ }^{\circ} \mathrm{C}$ and once the final temperature was achieved the furnace was left for dwelling for one hour.

The surface morphology was observed with an Olympus BX51 optical microscope using a $100 \times$ objective. The crystal structure was studied by using a Panalytical X'Pert PRO MPD $\mathrm{X}$-Ray diffractometer (Ni filtered $\mathrm{Cu} \mathrm{K}_{\alpha}$ radiation at $40 \mathrm{kV}$ and $40 \mathrm{~mA}$ ) operated in the $\theta-2 \theta$ mode. The background was subtracted using the Sonneveld method [35].

The composition of the films was investigated in transmission mode utilising a Nicolet iS10 FTIR spectrometer from Thermo Scientific with an optimised spectral range of $7800-350 \mathrm{~cm}^{-1}$ and a resolution of $0.4 \mathrm{~cm}^{-1}$.

The magnetic measurements were conducted with a Quantum Design MPMS-7 SQUID (superconducting quantum interference device) magnetometer. The films were deposited with a stripe shadow mask $\left(4 \times 90 \mathrm{~mm}^{2}\right)$ on flexible Kapton foil and rolled into a tube as reported by Heutz et al. [28]. Therefore the size of the Kapton foil was chosen to be larger than the scanning length of the coils in the SQUID to compensate for any background signals that could occur from the substrate.

\section{Acknowledgements}

We thank R. Sweeney and Dr R. Thorogate for help with XRD and SQUID measurements, respectively. We thank the Research Council UK and the Engineering and Physical Sciences Research Council (EPSRC, grants number EP/ F039948/1 and EP/F04139X/1) for financial support. P. R. and S. H. thank Kurt J. Lesker Company and Imperial College London for funding.

\section{References}

1. Forrest, S. R. Nature 2004, 428, 911-918. doi:10.1038/nature02498

2. Rocha, A. R.; García-Suárez, V. M.; Bailey, S. W.; Lambert, C. J.; Ferrer, J.; Sanvtio, S. Nat. Mater. 2005, 4, 335-339. doi:10.1038/nmat1349

3. Bao, Z.; Lovinger, A. J.; Dodabalapur, A. Appl. Phys. Lett. 1996, 69, 3066-3068. doi:10.1063/1.116841

4. Serri, M.; Wu, W.; Fleet, L. R.; Harrison, N. M.; Hirjibehedin, C. F.; Kay, C. W. M.; Fisher, A. J.; Aeppli, G.; Heutz, S. Nat. Commun. 2014, 5, 3079. doi:10.1038/ncomms4079

5. Eguchi, K.; Nanjo, C.; Awaga, K.; Tseng, H.-H.; Robaschik, P.; Heutz, S. Phys. Chem. Chem. Phys. 2016, 18, 17360-17365. doi:10.1039/C6CP01932C

6. Jiang, J., Ed. Functional Phthalocyanine Molecular Materials; Structure and Bonding, Vol. 135; Springer: Berlin, Germany, 2010 doi:10.1007/978-3-642-04752-7
7. Bayliss, S. M.; Heutz, S.; Rumbles, G.; Jones, T. S. Phys. Chem. Chem. Phys. 1999, 1, 3673-3676. doi:10.1039/a904089g

8. Heutz, S.; Bayliss, S. M.; Middleton, R. L.; Rumbles, G.; Jones, T. S. J. Phys. Chem. B 2000, 104, 7124-7129. doi:10.1021/jp0000836

9. Yim, S.; Heutz, S.; Jones, T. S. J. Appl. Phys. 2002, 91, 3632-3636. doi:10.1063/1.1446218

10. Miyoshi, H.; Ohya-Nishiguchi, H.; Deguchi, Y. Bull. Chem. Soc. Jpn. 1973, 46, 2724-2728. doi:10.1246/bcsj.46.2724

11. Mitra, S.; Gregson, A. K.; Hatfield, W. E.; Weller, R. R. Inorg. Chem. 1983, 22, 1729-1732. doi:10.1021/ic00154a007

12. Barraclough, C. G.; Martin, R. L.; Mitra, S.; Sherwood, R. C. J. Chem. Phys. 1970, 53, 1638-1642. doi:10.1063/1.1674236

13. Yamada, H.; Shiamda, T.; Koma, A. J. Chem. Phys. 1998, 108, 10256-10261. doi:10.1063/1.476486

14. Wu, W.; Kerridge, A.; Harker, A. H.; Fisher, A. J. Phys. Rev. B 2008, 77, 184403. doi:10.1103/PhysRevB.77.184403

15. Tseng, H.-H.; Serri, M.; Harrison, N. M.; Heutz, S. J. Mater. Chem. C 2015, 3, 8694-8699. doi:10.1039/C5TC01778E

16. Gentry, K. P.; Gredig, T.; Schuller, I. K. Phys. Rev. B 2009, 80, 174118. doi:10.1103/PhysRevB.80.174118

17. Brown, C. J. J. Chem. Soc. A 1968, 2488-2493. doi:10.1039/J19680002488

18. Figgs, B. N.; Mason, R.; Williams, G. A. Acta Crystallogr., Sect. B 1980, 36, 2963-2970. doi:10.1107/S056774088001062X

19. Scherrer, P. Nachr. Ges. Wiss. Goettingen, Math.-Phys. KI. 1918, 2, 98-100.

20. Hoshino, A.; Takenaka, Y.; Miyaji, H. Acta Crystallogr., Sect. B 2003, B59, 393-403. doi:10.1107/S010876810300942X

21. Robaschik, P.; Siles, P. F.; Bülz, D.; Richter, P.; Monecke, M.; Fronk, M.; Klyatskaya, S.; Grimm, D.; Schmidt, O. G.; Ruben, M.; Zahn, D. R. T.; Salvan, G. Beilstein J. Nanotechnol. 2014, 5, 2070-2078. doi:10.3762/bjnano.5.215

22. Khatkale, M. S.; Devlin, J. P. J. Chem. Phys. 1979, 70, 1851-1859. doi:10.1063/1.437662

23. Rückerl, F.; Mahns, B.; Dodbiba, E.; Nikolis, V.; Herzig, M.; Büchner, B.; Knupfer, M.; Hahn, T.; Kortus, J. J. Chem. Phys. 2016, 145, 114702. doi:10.1063/1.4962578

24. Seoudi, R.; El-Bahy, G. S.; El Sayed, Z. A. J. Mol. Struct. 2005, 753, 119-126. doi:10.1016/j.molstruc.2005.06.003

25. Kendall, D. N. Anal. Chem. 1953, 25, 382-389. doi:10.1021/ac60075a002

26. Nath, A.; Kopelev, N.; Tyagi, S. D.; Chechersky, V.; Wie, Y. Mater. Lett. 1993, 16, 39-44. doi:10.1016/0167-577X(93)90180-6

27. Awaga, K.; Maruyama, Y. Phys. Rev. B 1991, 44, 2589-2594. doi:10.1103/PhysRevB.44.2589

28. Heutz, S.; Mitra, C.; Wu, W.; Fisher, A. J.; Kerridge, A.; Stoneham, M.; Harker, T. H.; Gardener, J.; Tseng, H.-H.; Jones, T. S.; Renner, C.; Aeppli, G. Adv. Mater. 2007, 19, 3618-3622. doi:10.1002/adma.200701458

29. Barraclough, C. G.; Gregson, A. K.; Mitra, S. J. Chem. Phys. 1974, 60, 962-968. doi:10.1063/1.1681174

30. Taguchi, Y.; Miyake, T.; Margadonna, S.; Kato, K.; Prassides, K.; Iwasa, Y. J. Am. Chem. Soc. 2006, 128, 3313-3323. doi:10.1021/ja0582657

31. Taguchi, Y.; Miyake, T.; Margadonna, S.; Kato, K.; Prassides, K.; Iwasa, Y. J. Magn. Magn. Mater. 2007, 310, 1229-1230. doi:10.1016/j.jmmm.2006.10.733

32. Wang, Z.; Seehra, M. S. J. Phys.: Condens. Matter 2016, 28, 136002. doi:10.1088/0953-8984/28/13/136002

33. Moriya, T. Phys. Rev. 1960, 117, 635. doi:10.1103/PhysRev.117.635 
34. Lever, A. B. P. J. Chem. Soc. 1965, 1821-1829. doi:10.1039/jr9650001821

35. Sonneveld, E. J.; Visser, J. W. J. Appl. Crystallogr. 1975, 8, 1-7. doi:10.1107/S0021889875009417

\section{License and Terms}

This is an Open Access article under the terms of the Creative Commons Attribution License

(http://creativecommons.org/licenses/by/4.0), which permits unrestricted use, distribution, and reproduction in any medium, provided the original work is properly cited.

The license is subject to the Beilstein Journal of

Nanotechnology terms and conditions:

(http://www.beilstein-journals.org/bjnano)

The definitive version of this article is the electronic one which can be found at:

doi:10.3762/bjnano.8.146 